ORIGINAL ARTICLE

\title{
Status of the p53, p16, RB1, and HER-2 genes and chromosomes 3, 7, 9, and 17 in advanced bladder cancer: correlation with adjacent mucosa and pathological parameters
}

\author{
M Gallucci, F Guadagni, R Marzano, C Leonardo, R Merola, S Sentinelli, E M Ruggeri, R Cantiani, \\ I Sperduti, F de la Iglesia Lopez, A M Cianciulli
}

J Clin Pathol 2005;58:367-371. doi: 10.1136/icp.2004.021154

See end of article for authors' affiliations .....................

Correspondence to: Dr A M Cianciulli, Clinical Pathology, Regina Elena Cancer Institute, IFO, Via Elio Chianesi, 53, 00144 Rome, Italy; cianciulli@ifo. it

Accepted for publication 15 September 2004

\begin{abstract}
Aims: To evaluate a panel of well known genetic alterations for frequency of changes in bladder cancer that could be considered genomic instability determinants or adjunctive prognostic predictors.

Methods: Fluorescence in situ hybridisation analysis was performed to evaluate chromosomes 3, 7, 9, and 17 and the 9p21 (p16), 17p13.1 (p53), 13q14 (RB1), and 17q11.2 (HER-2) chromosomal loci in 48 muscle invasive bladder cancer specimens and the adjacent normal mucosa.

Results: There were significant differences between the frequency of chromosome 7 monosomy/polysomy and 17 monosomy in the two groups (tumours and adjacent mucosa) ( $p=0.004, p=0.037$, and $p=0.015$, respectively). There were no differences in the frequency of gene deletions between tumours and the adjacent mucosa. 17q1 1.2 amplification was found in $14.5 \%$ of tumours examined, but not in the non-malignant epithelium. Chromosome 3, 7, and 17 monosomy and the RB1 heterozygous deletion were significantly associated with stage T3-4 $(p=0.03, p=0.04, p=0.04$, and $p=0.03$, respectively). Conclusions: These results demonstrate the importance of chromosomes 3, 7, and 17 and gene alterations in bladder cancer progression, highlighting their usefulness as prognostic markers. Larger studies with longterm follow up of these patients are needed to determine the validity and clinical relevance of these genetic findings, and molecular prognostic markers should be incorporated into phase II and III trials to define their roles in predicting clinical outcome.
\end{abstract}

$\mathrm{T}$ here are convincing data to support the hypothesis that a large number of genetic events are involved in the aetiology, progression, and response to treatment of bladder cancer. ${ }^{1}$ Recently, a large body of information has been generated with regard to the growth regulatory pathway through which the p53 and retinoblastoma $(\mathrm{Rb})$ proteins work, including the MDM2, CDKN1A (p2l), E2F, and MYCL genes. ${ }^{2}$ Moreover, it has been shown that the genesis and/or progression of bladder cancer is a consequence of genetic instability, and that chromosomes 3, 7, 9, and 17 are frequently involved in uroepithelial oncogenesis. ${ }^{3}{ }^{4}$ HER-2 gene amplification has been shown to be associated with a poor prognosis in bladder cancer. ${ }^{5}$

"The identification of patients at increased risk of progression is an important goal in bladder cancer research because such patients will be candidates for newer treatments and follow up strategies"

In our study, we analysed deletions of 9p21 (p16), 17p13.1 (p53), and 13q14 (RB1) in 48 bladder cancer specimens and the adjacent normal mucosa. In the same specimens, the status of the 3, 7, 9, and 17 chromosomes and of the HER-2 gene was examined. The genetic evaluation was determined using fluorescence in situ hybridisation (FISH). The preliminary part of our investigation analysed the frequency of chromosomal alterations, gene amplification, and deletion in bladder cancer and the surrounding mucosa to evaluate general genetic instability in the entire transitional epithelium. In the second part of our study, we concentrated on patients with advanced disease to evaluate the role of molecular markers that could potentially have important prognostic implications by complementing the standard histopathological staging system. The identification of patients at increased risk of progression is an important goal in bladder cancer research because such patients will be candidates for newer treatments and follow up strategies.

\section{MATERIALS AND METHODS}

Patient material and histopathological diagnosis

Our study included 48 patients who underwent radical cystectomy, pelvic lymphadenectomy, and urinary reconstruction for muscle invasive $(\mathrm{n}=42)$ or recurrent high grade superficial $(n=6)$ bladder cancer. Fresh tumour samples were used for touch imprinting preparations. Histological diagnosis and tumour grading were performed by an experienced uropathologist according to the UICC TNM $^{6}$ and 1998 World Health Organisation classifications. ${ }^{7}$ Specimens were classified as follows: Tl $(\mathrm{n}=6)$, T2a $(\mathrm{n}=5)$, T2b $(\mathrm{n}=8)$, T3a $(\mathrm{n}=8)$, T3b $(\mathrm{n}=10)$, and T4a $(\mathrm{n}=11)$ tumours all belonged to grade 3 . Nineteen of the 48 tumours had lymph node involvement. Informed consent was obtained from all patients and tissue sampling was approved by the ethics committee of the National Cancer Institute, Rome, Italy. In addition, 20 tissue samples, which were considered non-malignant by conventional histology, were also taken from adjacent sites on the urothelium. As a control population, normal bladder tissue samples from

Abbreviations: CEP, chromosome enumeration probe; FISH, fluorescence in situ hybridisation; LSI, locus specific identifier; Rb, retinoblastoma 
Table 1 Mean percentage of cells after hybridisation with specific probes for chromosomes 3, 7, 9, and 17 and $13 q 14$ (RB1 gene) in control cases

\begin{tabular}{|c|c|c|c|c|c|c|c|c|c|c|}
\hline & \multicolumn{2}{|c|}{ Chromosome 3} & \multicolumn{2}{|c|}{ Chromosome 7} & \multicolumn{2}{|c|}{ Chromosome 9} & \multicolumn{2}{|c|}{ Chromosome 17} & \multirow{2}{*}{$\begin{array}{l}\mathrm{HO} \\
\mathrm{RB1}\end{array}$} & \multirow{2}{*}{$\begin{array}{l}\mathrm{HE} \\
\mathrm{RB} 1\end{array}$} \\
\hline & $M$ & $\mathbf{P}$ & $M$ & $\mathbf{P}$ & $M$ & $\mathbf{P}$ & $M$ & $\mathbf{P}$ & & \\
\hline Mean & 2.20 & 0.80 & 3.42 & 1.0 & 1.40 & 0.70 & 11.1 & 1.50 & 0.00 & 9.30 \\
\hline SD & 2.91 & 1.90 & 4.12 & 2.54 & 2.66 & 1.16 & 4.6 & 1.94 & 0.00 & 4.95 \\
\hline
\end{tabular}

patients undergoing prostatectomy, as a result of benign pathology, were used.

\section{FISH analysis}

Touch preparations were reviewed by a pathologist to verify the adequacy of the cellular components. The FISH analysis procedure has been described previously. ${ }^{4}$ The fluorescent labelled probes for FISH assay, specific for the centromeric region of chromosomes 3 (D3Z1), 7 (D7Z1), 9 (D9Z5), and 17 (D17Z1) (Vysis Inc, Downers Grove, Illinois, USA) were used for specific ploidy detection. We also used specific probes for pl6 (9p21), p53 (17p13.1), and RBl (13q14) (Vysis) to assess the deletion (not mutation) of these loci. The status of the HER-2 gene was evaluated by the HER-2 DNA probe kit (Vysis). We also applied chromosome enumeration probes (CEP) 9 and 17 to adjust for the effects of aneuploidy and to establish the presence of amplification and/or deletion of HER-2, p53, and 9p21. Copy numbers for centromeres and specific gene regions were counted in at least 100 nonoverlapping cells. Some samples were not evaluated for all variables for technical reasons.

\section{Statistical analysis}

The Mann-Whitney $U$ test was used to assess differences between frequencies of genetic aberrations in the two groups examined (tumours and distal mucosa). The $\chi^{2}$ test was used to evaluate the association of genetic markers with pathological stage and lymph node status, and significance was set at $\mathrm{p}<0.05$.

\section{RESULTS}

Chromosome 3, 7, 9, and 17 and LSI (17q11.2, 9p21, $17 p 13.1$, and $13 q 14)$ status in normal and neoplastic bladder tissue

In this first evaluation, we performed a control FISH assay to establish the hybridisation patterns in bladder samples from 15 patients undergoing prostatectomy (table 1).

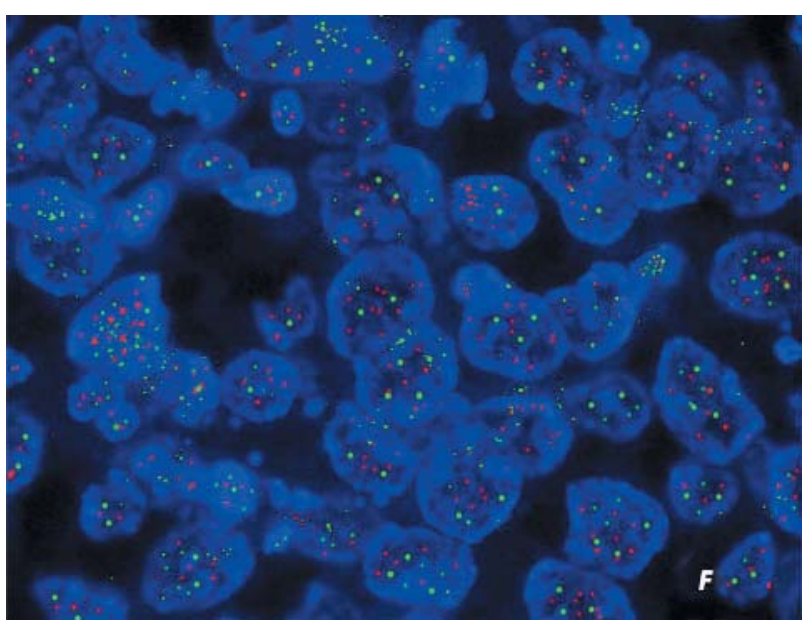

Figure 1 Fluorescence in situ hybridisation image: CEP 17 (green) and LSI 17q11.2-q12 (red) in a case with chromosome 17 polysomy and a ratio (gene to centromeric signals) $>2$.

Tumours and distal epithelial samples were determined to be monosomic and/or polysomic for chromosomes 3, 7, 9, and 17 and deleted for the RBl gene when the dominant population exceeded the mean +3 SD of any of the signal categories seen in the control group. In addition, we used the locus specific identifier (LSI) 13 probe (Vysis) to distinguish between failure of hybridisation and deletion of the RBI sequence. Homozygous deletion of the RBl gene was present when no RBl and two LSI 13 signals were seen, whereas heterozygous deletion was present when one RBl and two LSI 13 signals were seen. The control for aneuploidy of chromosome 17, where the HER-2 and p53 genes are located, was an $\alpha$ satellite centromere probe, which was co-hybridised with the HER-2 and p53 genes. The probes for chromosome 9

Table 2 Comparison between tumour and normal adjacent mucosa for all examined genetic markers

\begin{tabular}{llll}
\hline & Tumour $(\mathbf{n = 4 8 )}$ & Distal mucosa $(\mathbf{n = 2 0 )}$ & $\mathbf{p}$ Value \\
\hline Chromosome 3 monosomy & $21(43.7)$ & $7(35.0)$ & $\mathrm{NS}$ \\
Chromosome 3 polysomy & $19(39.5)$ & $4(20.0)$ & $\mathrm{NS}$ \\
Chromosome 7 monosomy & $21(43.7)$ & $1(5.0)$ & 0.004 \\
Chromosome 7 polysomy & $21(43.7)$ & $3(15.0)$ & 0.037 \\
Chromosome 9 monosomy & $33(68.7)$ & $11(55.0)$ & $\mathrm{NS}$ \\
Chromosome 9 polysomy & $10(20.8)$ & $1(5.0)$ & $\mathrm{NS}$ \\
Chromosome 17 monosomy & $18(37.5)$ & $1(5.0)$ & 0.015 \\
Chromosome 17 polysomy & $20(41.6)$ & $5(25.0)$ & $\mathrm{NS}$ \\
p16 HO & $4(8.3)$ & $0(0.0)$ & $\mathrm{NS}$ \\
p16 HE & $32(66.6)$ & $17(85.0)$ & $\mathrm{NS}$ \\
p53 HO & $4(8.3)$ & $1(5.0)$ & $\mathrm{NS}$ \\
p53 HE & $27(56.2)$ & $17(85.0)$ & $\mathrm{NS}$ \\
RB1 HO & $6(12.5)$ & $2(10.0)$ & $\mathrm{NS}$ \\
RB1 HE & $39(81.2)$ & $15(75.0)$ & $\mathrm{NS}$ \\
HER-2 amplification & $7(14.5)$ & $0(0.0)$ & $\mathrm{N}$ \\
\hline
\end{tabular}

Numbers in parentheses represent percentages. Significance was measured using the Mann-Whitney test (nonparametric test).

$\mathrm{HE}$, heterozygous deletion; $\mathrm{HO}$, homozygous deletion; NS, not significant. 


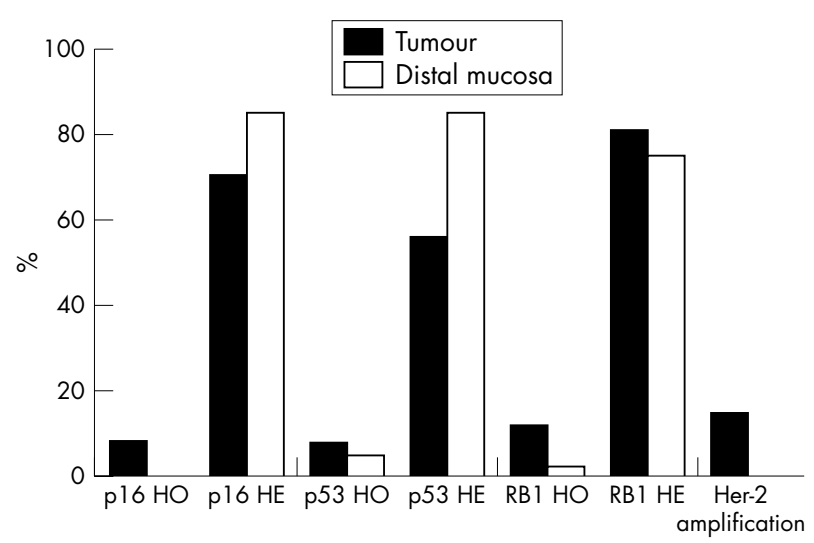

Figure 2 Locus specific identifier probe distribution in the samples. HE, heterozygous deletion; $\mathrm{HO}$, homozygous deletion.

(CEP 9 and LSI 9p21) were chosen to detect monosomy/ polysomy and losses of the pl6 gene, respectively. We used a ratio of more than two oncogene signals/centromere to define HER-2 gene amplification. We used ratios of 17p13.1 : CEP 17 and 9p21: CEP 9 lower than 0.5 as a measure of homozygous deletion, whereas heterozygous deletion was determined for ratios between 0.5 and 1 . Table 2 compares tumour and normal adjacent mucosa for all examined genetic markers, based on established criteria.

Monosomy and polysomy of the evaluated chromosomes ranged from $20.8 \%$ (chromosome 9 polysomy) to $68.7 \%$ (chromosome 9 monosomy) in the tumours and from 5.0\% (chromosome 7 and 17 monosomy and chromosome 9 polysomy) to $55.0 \%$ (chromosome 9 monosomy) in the adjacent mucosa. A significant difference was seen only for chromosomes 7 and 17. Most of the tumours and adjacent mucosa $(68.7 \%$ and $55.0 \%$, respectively) showed monosomy of chromosome 9 (table 2). As illustrated in table 2, FISH analysis performed using LSI probes revealed no differences in the frequency of deletions between tumour samples and the surrounding mucosa. The highest percentage of homozygous deletion of neoplastic informative cases was found at the 13q14 (RBl) locus (12.5\%). Homozygous deletion of the $9 \mathrm{p} 21$ (p16) and 17p13 (p53) loci was present in $8.6 \%$ of the patients. Heterozygous deletion was more frequent than homozygous deletion in cancer samples, ranging from $56.2 \%$ (17p13) to $81.2 \%$ (13q14). Even though the difference is not significant, HER-2 amplification was found in seven tumour specimens $(14.5 \%)$, whereas the adjacent mucosa did not show amplification. Five HER-2 amplified tumours had concomitant chromosome 17 polysomy (fig 1). Figure 2 shows the distribution of the LSI probes in the samples.

\section{Association between genetic alterations and pathological characteristics}

In the second part of our study, contingency table 3 and the $\chi^{2}$ test were used to evaluate the association between the pathological characteristics and genetic alterations in the 48 samples. In this part of the study, the discriminative power of heterozygous deletion and chromosomal aberrations was considered to be the median value of the percentage alteration. With regard to homozygous deletion, the discriminative power was its presence or absence.

Chromosome 3, 7, and 17 monosomy and RB heterozygous deletion were significantly associated with T3-4 stage $(\mathrm{p}=0.03, \mathrm{p}=0.04, \mathrm{p}=0.04$, and $\mathrm{p}=0.03$, respectively).

In some cases (underlined in table 3), when chromosomal and gene characteristics were compared with T3-4 stage and lymph nodal involvement, a positive trend was found even though the results were not significant.

\section{DISCUSSION}

Our evaluation of chromosomes 3, 7, 9, and 17 and the p53, pl6, RB, and HER-2 genes in human bladder neoplasia focused on specific issues, namely: (1) the detection and comparison of multiple genetic alterations identified in bladder cancer and in normal urothelium, and (2) the association of genetic alterations with clinicopathological characteristics. We found chromosomal numerical aberrations in all specimens analysed. Nevertheless, when malignant and non-malignant cells were compared, significant differences were seen only for chromosomes 7 and 17. These results, in agreement with those of Cordon-Cardo et al, ${ }^{8}$ show that the T3-4 stages are significantly associated with chromosome 3, 7, and 17 monosomy.

Most tumours and normal urothelium showed chromosome 9 monosomy, with mean percentages of $71.2 \%$ and $55.0 \%$, respectively. These data, according to a recent study performed by our group on superficial bladder cancer, ${ }^{4}$ indicate that this alteration is ubiquitous, and is not related to specific pathogenetic subsets, histological grade, or invasive phenotype. In addition, no significant differences in 9p21 deletion were found between malignant and nonmalignant cells.

The clinical relevance of p53 suppressor gene mutation is a controversial issue in bladder cancer. Alterations in the p53 and RB tumour suppressor genes are an important component in the development of bladder cancer, but the downstream pathways that contribute to urothelial transformation are not completely defined..$^{10}$ We found no significant difference between cancer specimens and the distal mucosa with regard to p53 deletion. The presence of allelic losses of the TP53 markers in cystectomy specimens and in areas of urothelium that are considered benign has been demonstrated by conventional histology. ${ }^{11}{ }^{12}$ These data, together with the results of our study, support the hypothesis that many normal appearing areas of the bladder are genetically altered in patients with bladder cancer.

\section{"Our study strongly confirms the importance of chromo- some 17 polysomy in HER-2 amplification detection"}

Inactivation of the RBl gene is a common event in bladder cancer and it has been associated with higher grade, invasive stage tumours, and decreased survival. ${ }^{13}{ }^{14}$ We found that the histopathological stage was significantly associated with RBl heterozygous deletion. In addition, a higher proportion of neoplastic samples had RBl deletion, both for homozygous (11.0\%) and for heterozygous $(86.0 \%)$ deletion.

Emerging data on the use of HER-2 amplification as a prognostic marker and/or therapeutic target (Trastuzumab) in breast cancer ${ }^{15}$ stimulated our interest in assessing the rate of HER-2 amplification in these specimens, and we were also encouraged by the cancer and leukemia group B, which has started a trial of single agent Trastuzumab in patients with previously treated advanced bladder disease. ${ }^{16}$ Studies of HER-2 overexpression and amplification have shown varying results in bladder cancer, as a result of the application of different laboratory techniques. ${ }^{4}$ Sauter et al found amplification in 10 of 141 bladder tumours, but only in those with aneusomy of chromosome $17 .{ }^{17}$ Chromosome 17 polysomy was detected in five of seven of our amplified tumours. Polysomy of chromosome 17 in bladder cancer occurs independently of tumour polyploidy. ${ }^{18}$ Our study strongly confirms the importance of chromosome 17 polysomy in HER-2 amplification detection. 


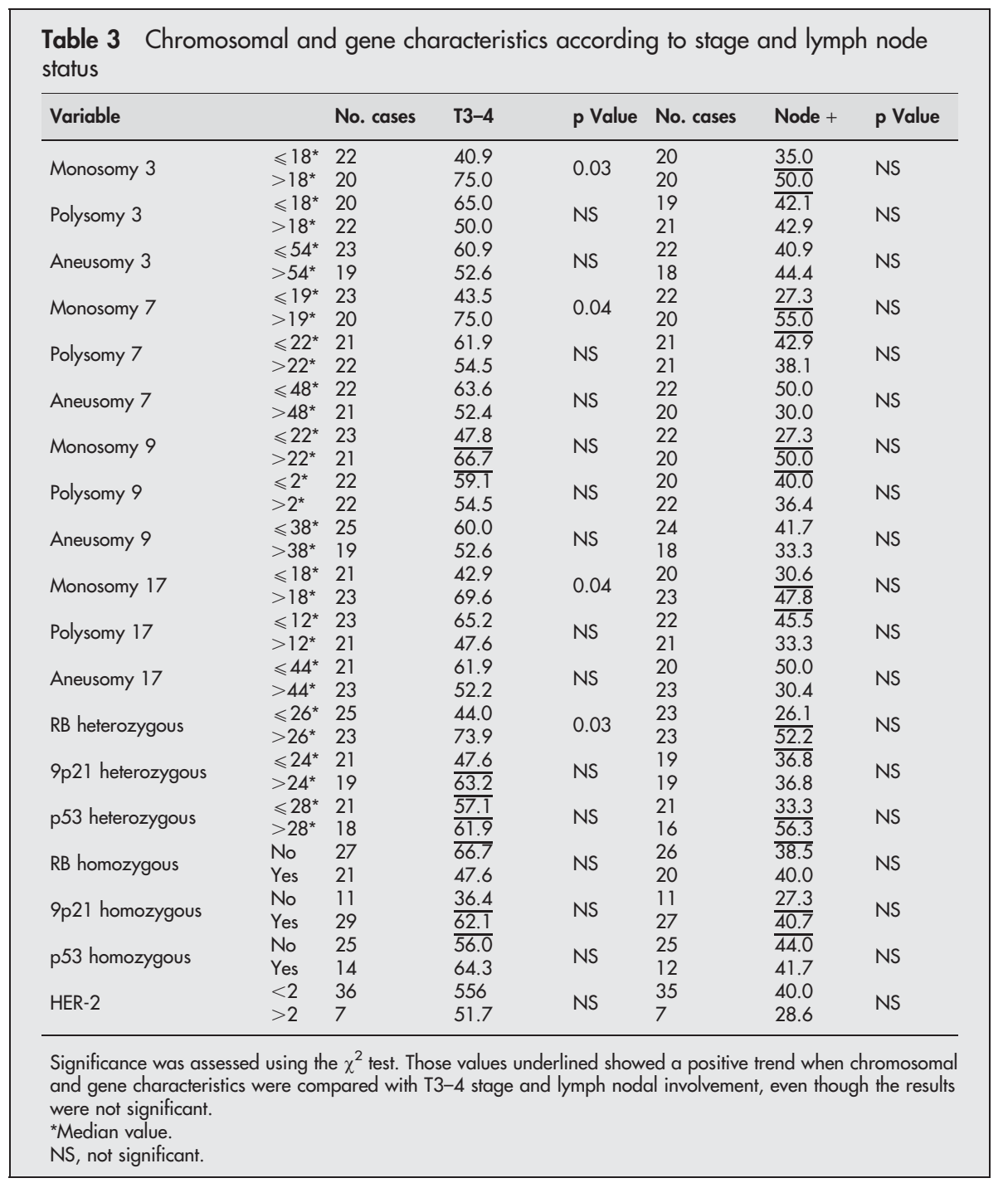

The results of our investigation highlight: (1) the presence of general genetic instability in the entire transitional epithelium and a close genetic relation between tumours and the adjacent mucosa; and (2) that the status of chromosomes 7 and 17 and the RBl gene could be useful genetic markers to complement the standard histopathological staging system and to identify patients at risk of progression. Because bladder cancer progression is undoubtedly associated with particular somatic genetic alterations, individual patients could be characterised by defining any significant genetic aberrations in the tumour obtained during

\section{Take home messages}

- Alterations in chromosome 3, 7, and 17 ploidy and RB1 gene alterations were found in bladder cancer

- Chromosome 3, 7, and 17 monosomy and RB1 heterozygous deletion might be potentially useful intermediate biomarkers to detect those patients at high risk of progression who may benefit from particular and innovative therapeutic interventions

- Larger, longterm studies with follow up are needed to assess the validity and clinical relevance of these genetic findings cystectomy. We propose that chromosome 3, 7, and 17 monosomy and RBl heterozygous deletion should be considered potentially useful intermediate biomarkers to detect patients at high risk of progression who may benefit from particular and innovative therapeutic interventions. Each of these parameters individually and together could improve the understanding of this disease and contribute to categorising patients with advanced bladder cancer. Only larger studies with longterm follow up will determine the usefulness of this observation.

\section{ACKNOWLEDGEMENTS}

We thank Mrs P Franke for the formal English revision of the manuscript and Mrs P Canalini for technical support. Supported by Italian Ministry of Health Project "Trattamento del carcinoma muscolo invasivo della vescica. Fattori prognostici e predittivi della risposta alla chemioterapia adiuvante".

\section{Authors' affiliations}

M Gallucci, C Leonardo, R Cantiani, Department of Urology, Regina Elena Cancer Institute, Via Elio Chianesi, 53, 00144 Rome, Italy F Guadagni, R Marzano, R Merola, F de la I Lopez, A M Cianciulli, Department of Clinical Pathology, Cytogenetic Unit, Regina Elena Cancer Institute

S Sentinelli, Department of Pathology, Regina Elena Cancer Institute E M Ruggeri, Department of Oncology, Regina Elena Cancer Institute I Sperduti, Biostatistic Unit, Regina Elena Cancer Institute 


\section{REFERENCES}

1 Kausch I, Bohle A. Molecular aspects of bladder cancer III. Prognostic markers of bladder cancer. Eur Urol 2002;41:15-29.

2 Stein JP, Grossfeld GD, Ginsberg DA, et al. Prognostic markers in bladder cancer: a contemporary review of the literature. J Urol 1998;160:645-59.

3 Knowles MA. The genetics of transitional cell carcinoma: progress and potential clinical application. BJU Int 1989;84:412-27.

4 Cianciulli AM, Leonardo C, Guadagni F, et al. Genetic instability in superficial bladder cancer and adjacent mucosa: an interphase cytogenefic study. Hum Pathol 2003;34:214-21.

5 Miyamoto H, Kubota Y, Noguchi S, et al. C-ERBb-2 gene amplification as a prognostic marker in human bladder cancer. Urology 2000;55:679-83.

6 UICC. TNM classification of malignant tumors, 6th ed. New York: Wiley-Liss, 2002.

7 Epstein JI, Amin MB, Reuter VR, et al. The World Health Organization/ International Society of Urological Pathology consensus classification of urothelial (transitional cell) neoplasms of the urinary bladder. Bladder consensus conference committee. Am J Surg Pathol 1998;22:1435-48.

8 Cordon-Cardo C, Sheinfeld J, Dalbagni G. Genetic studies and molecular markers of bladder cancer. Semin Surg Oncol 1997;13:319-27.

9 Cote RJ, Esrig D, Groshen S, et al. p53 and treatment of bladder cancer. Nature 1997;385:123-4.

10 Shariat SF, Tokunaga $\mathrm{H}$, Zhou $\mathrm{JH}$, et al. p53, p21, pRB, and $\mathrm{p} 16$ expression predict clinical outcome in cystectomy with bladder cancer. $J$ Clin Oncol 2004;22:1014-24.
11 Chaturvedi V, Li L, Hodges S, et al. Superimposed histologic and genetic mapping of chromosome 17 alterations in human urinary bladder neoplasia. Oncogene 1997; 14:2059-70.

12 Simon R, Eltze E, Schafer KL, et al. Cytogenetic analysis of multifocal bladder cancer supports a monoclonal origin and intraepithelial spread of tumor cells. Cancer Res 2001;61:355-62.

13 Sourvinos G, Kazanis I, Delakas D, et al. Genetic detection of bladder cancer by microsatellite analysis of $\mathrm{p} 16, \mathrm{RB} 1$ and $\mathrm{p} 53$ tumor suppressor genes. J Urol 2001;165:249-52.

14 Cordon-Cardo C, Wartinger D, Petrylak D, et al. Altered expression of the retinoblastoma gene product: prognostic indicator in bladder cancer. I Natl Cancer Inst 1992;84:1251-6.

15 Pegram MD, Lipton A, Hayes DF, et al. Phase II study of receptor-enhanced chemosensitivity using recombinant humanized anti-p185 HER2/neu monoclonal antibody plus cisplatin in patients with HER-2 overexpressing metastatic breast cancer refractory to chemotherapy treatment. J Clin Oncol 1998;16:2659-71.

16 Small EJ, Halabi S, Dalbagni G, et al. Overview of bladder cancer trials in the cancer and leukemia group B. Cancer Suppl 2003;97:2090-8.

17 Sauter G, Moch H, Moore D, et al. Heterogeneity of erbB-2 gene amplification in bladder cancer. Cancer Res 1993;53:2199-203.

18 Latif Z, Watters AD, Dunn I, et al. HER2/neu gene amplification and protein overexpression in G3 pT2 transitional cell carcinoma of the bladder: a role for anti-HER2 therapy? Eur J Cancer 2004;40:56-63. 